\title{
Propiedades psicométricas del Patient Health Questionnaire (PHQ-9) en estudiantes de medicina en Lima, Perú.
}

\author{
Psychometric properties of the Patient Health Questionnaire (PHQ-9) in medical students, Lima, Peru. \\ Jeff Huarcaya-Victoria ${ }^{1,2, a}$, Raúl De Lama-Morán ${ }^{3, b}$, María Quiros ${ }^{4, ~ c, ~ J i m e n a ~ B a z a ́ n ~}{ }^{4, ~ c}$, Kevin López ${ }^{4, ~ c, ~}$ \\ Dáneri Lora ${ }^{4, c}$

\section{RESUMEN}

Se ha reportado que el Patient Health Questionnaire (PHQ-9) es una herramienta psicométricamente válida y confiable aun cuando, evaluado mediante análisis factoriales, no queda claro si una estructura de uno o dos factores es la mejor. Objetivo: Evaluar las propiedades psicométricas y la estructura factorial del PHQ-9 en estudiantes de medicina de una universidad del Perú. Material y Métodos: Investigación instrumental en 200 estudiantes de medicina. Un análisis factorial exploratorio (AFE) permitió evaluar la estructura interna del PHQ-9, sometida luego a un análisis factorial confirmatorio (AFC) de tres modelos. Resultados: Se encontró una adecuada consistencia interna (alfa de Cronbach $=0,903)$. Mediante el AFC se encontraron adecuados índices de ajuste en el modelo bifactorial $\left(x^{2}(17)=\right.$ 26,451, $p=0,067$; $\mathrm{CFI}=0,991$; GFI $=0,969$; $\mathrm{RMSEA}=0,056$ ) en comparación con los modelos unidimensional y bidimensional. Conclusiones: El PHQ-9 es un instrumento con propiedades psicométricas adecuadas para su aplicación en estudiantes de medicina de una universidad del Perú. El AFC reveló que un modelo bifactorial que toma en cuenta tanto el factor general como factores latentes somáticos y cognitivo/afectivos, muestra un mejor ajuste.

PALABRAS CLAVE: Depresión; Patient Health Questionnaire-9; análisis factorial; Perú.

\section{SUMMARY}

The Patient Health Questionnaire (PHQ-9) is recognized as a psychometrically valid and reliable intrument; however, when assessed by means of a factor analysis method, it remains unclear whether a one- or two-factor structure is the best. Objective: To evaluate the psychometric properties and the factor structure of the PHQ-9 in medical students of a university in Peru. Material and Methods: Instrumental study in 200 medical students. An exploratory factor analysis (EFA) was performed to evaluate the internal structure of the PHQ-9, followed by a confirmatory factor analysis (CFA) of three models. Results: An adequate internal consistency was found (Cronbach's alpha $=0,903$ ).

1 Centro de Investigación en Salud Pública, Facultad de Medicina, Universidad de San Martín de Porres. Lima, Perú.

2 Departamento de Psiquiatría, Servicio de Psiquiatría de Adultos, Hospital Nacional Guillermo Almenara Irigoyen, EsSalud. Lima, Perú.

3 Centro de Investigación en Educación Médica, Facultad de Medicina, Universidad de San Martín de Porres, Lima, Perú.

4 Facultad de Medicina, Universidad de San Martín de Porres. Lima, Perú.

a Médico psiquiatra, Jefe de la Unidad de Psiquiatría de Enlace.

b Psicólogo, Magíster en psicología clínica.

c Estudiante de medicina. 
Propiedades psicométricas del Patient Health Questionnaire (PHQ-9) en estudiantes de medicina en Lima, Perú.

Through the CFA, the bifactorial model $(x 2(17)=26,451, p=0,067$; CFI $=0,991$; GFI $=0,969$; RMSEA $=0,056)$ had a better adjustment than the unidimensional and bi-dimensional factor models. Conclusion: The PHQ-9 is an instrument with adequate psychometric properties for its application among medical students of a Peruvian university. The CFA revealed that a bifactorial model, in which both the general factor and latent somatic and cognitive/affective factors are taken into account, yields more fitting results.

KEYWORDS: Depression; Patient Health Questionnaire-9; factorial analysis; Peru.

\section{INTRODUCCIÓN}

La depresión es uno de los trastornos psiquiátricos más comunes en la población general del Perú. Según los reportes epidemiológicos del Instituto Nacional de Salud Mental Honorio Delgado - Hideyo Noguchi (INSM HD-HN), la prevalencia de vida de depresión en Lima y Callao es del $17,3 \%$, la cual es mayor en mujeres (21,6\%) respecto a varones $(12,6 \%)$ (1). Estas cifras suelen ser mayores en los estudiantes de medicina. En un reciente metanálisis se encontró una prevalencia de síntomas depresivos del $28 \%$ en estudiantes de medicina, de los cuales solo el 12,9\% buscó ayuda profesional (2). La presencia de síntomas depresivos en los estudiantes de medicina han sido relacionados con una disminución de la capacidad para manejar el estrés académico, retiros de la universidad, bajo rendimiento académico y en el peor de los casos, ideas o conductas suicidas (2). Por lo tanto, la búsqueda activa de los casos de depresión en dicha población es una actividad esencial para reducir el impacto de este problema de salud pública.

Para poder diagnosticar adecuadamente un trastorno depresivo es fundamental la realización de entrevistas clínicas por personas especializadas. No obstante, dichas entrevistas pueden ser prolongadas, además de no resultar adecuadas en entornos no clínicos. Es factible utilizar cuestionarios de fácil aplicación para poder realizar un tamizaje y seguimiento a grandes poblaciones, con lo cual se podría incrementar las tasas de detección de trastornos depresivos en los estudiantes de medicina.

De todas las escalas de tamizaje y seguimiento de los síntomas depresivos, el Patient Health Questionnaire (PHQ-9) es uno de los más utilizados en los últimos años, debido a la facilidad de su aplicación (3). Es una versión resumida de una sección del Primary Care Evaluation of Mental Disorders (PRIME-MD), el cual fue inicialmente desarrollado para detectar los cinco trastornos mentales más comunes en el primer nivel de atención: depresión, ansiedad, dependencia de alcohol, trastornos somatomorfos y trastornos de la alimentación (4). El PHQ-9 fue desarrollado con el propósito de establecer un diagnóstico provisional de depresión y determinar la severidad de los síntomas depresivos (3). Debido a su fácil uso, el PHQ-9 ha sido recomendado en guías de práctica clínica para valorar la severidad de los síntomas depresivos. Sin embargo, aunque se ha reportado que el PHQ-9 es una herramienta psicométricamente válida y confiable, a partir de los análisis factoriales no está claro si una estructura de uno o dos factores es la mejor (5).

En un juicio de expertos peruanos se concluyó que, luego de realizar algunos cambios en la traducción al español, el PHQ-9 podría ser un instrumento válido para diagnosticar un trastorno depresivo y poder ser aplicado en el Perú (6). Posteriormente, en el contexto peruano, el PHQ-9 fue validado en mujeres embarazadas (7) y pacientes con tuberculosis (8), en los cuales se encontró adecuadas propiedades psicométricas. No obstante, hasta donde tenemos conocimiento, no existen estudios de análisis factorial confirmatorio (AFC) del PHQ-9 en estudiantes de medicina peruanos.

Este estudio tuvo como objetivo el analizar las propiedades psicométricas y realizar un $\mathrm{AFC}$ de tres modelos (unidimensional, bidimensional, bifactorial) del PHQ-9 en una muestra de estudiantes de medicina de una universidad privada del Perú. Así, este trabajo podría servir como antecedente para futuros estudios que requieran utilizar el PHQ-9, con lo que esperamos llenar un vacío teórico respecto a las propiedades psicométricas de esta escala en la población de estudiantes universitarios peruanos.

\section{MATERIAL Y MÉTODOS}

Se trató de un estudio instrumental, observacional y transversal, que buscó explorar las propiedades psicométricas del PHQ-9 en una muestra de estudiantes de medicina. La población de estudio estuvo conformada por alumnos de medicina de 1er a 6to 
año de la Facultad de Medicina de la Universidad de San Martín de Porres (FMH-USMP). La recolección de datos se realizó de forma virtual durante el mes de julio. Para el cálculo del tamaño de muestra para el AFC utilizamos el criterio de cantidad absoluta de casos $(n=200)$ propuesto por Hair et al. (9), quienes consideran que una muestra de 200 es ideal para proporcionar una base sólida para la estimación en los AFC. Se enviaron los instrumentos de evaluación de forma virtual a todos los alumnos de la FMH-USMP de 1er a 6to año durante el periodo académico 2019-I. Luego se procedió a seleccionar de forma aleatoria a 200 alumnos del total que decidieron participar.

\section{Medición de los síntomas depresivos}

Se utilizó el PHQ-9, el cual es una escala autoadministrada. Consta de 9 ítems, los cuales se encuentran directamente relacionados con la sintomatología depresiva durante las dos semanas previas a la autoaplicación de la escala. Cada ítem se puntúa según una escala Likert que va desde 0 (ningún día) a 3 (casi todos los días). Los puntajes del PHQ-9 reflejan 5 categorías de severidad del trastorno depresivo: ninguna (0-4 puntos), leve (5-9 puntos), moderado (10-14 puntos), moderadamente severo (15-19 puntos) y severo (20-27 puntos). Respecto a sus propiedades psicométricas, en un estudio se encontró que tiene una sensibilidad y especificidad del $88 \%$ cuando el PHQ-9 $\geq 10$, además cuenta con una adecuada consistencia interna (alfa de Cronbach $=0,89$ ), un puntaje de prueba-respuesta de 0,84 , una concordancia de $84 \%$ y un área bajo la curva ROC de 0,95 (3). En estudios realizados en Latinoamérica se ha demostrado que el PHQ-9 es una herramienta válida y confiable para la detección de síntomas depresivos en diversos tipos de población $(10,11)$. Para este estudio se utilizó la versión modificada del PHQ-9 realizada por un grupo de expertos peruanos (6).

\section{Análisis de los datos}

Se realizaron técnicas de estadística descriptiva para todas las variables, reportando la media y desviación estándar $(X \pm \sigma)$, mínimo y máximo para las variables numéricas y análisis de frecuencias para las variables categóricas.

Se llevó a cabo un análisis de la validez de la PHQ-9, para lo cual primero se evaluó la viabilidad de realizar un análisis factorial exploratorio (AFE) mediante el criterio de adecuación factorial de KeiserMeyer-Olkin (KMO) y la prueba de esfericidad de
Barlett. El número de factores se determinó utilizando el criterio de autovalor $>1$ utilizando el método de análisis de componentes principales, y luego se ajustó mediante rotación Varimax con normalización de Kaiser. La fiabilidad se evaluó a través de un análisis de consistencia interna con alfa de Cronbach.

Posteriormente se realizó un AFC de los tres modelos. En el modelo unidimensional, cada ítem fue representado por un solo factor general. En el modelo bidimensional, los ítems se agruparon en dos factores latentes: somático $(3=$ problemas con el sueño, $4=$ fatiga, $5=$ cambios en el apetito) y cognitivo/afectivo $(1=$ anhedonia, 2 = ánimo deprimido, $6=$ sentimientos de inutilidad, 7 = problemas con la concentración, 8 = enlentecimiento/agitación, 9 = ideas suicidas). En el modelo bifactorial, utilizamos la agrupación propuesta por Doi et al. (12), quienes designaron los síntomas somáticos y cognitivos/afectivos como factores de grupo específicos, y la suma de los ítems como el factor general. Se siguió la guía propuesta por Boomsma (13) para el AFC. La identificación de posibles casos atípicos se realizó mediante la distancia de Mahalanobis, siendo el nivel de significancia el recomendado por Kline $(p<0,001)(14)$. La literatura sugiere remover los casos atípicos univariados y multivariados. El ajuste del modelo propuesto se estimó con el método de máxima verosimilitud (maximum likelihood, ML). Se usaron tres estadísticos de bondad de ajuste del modelo recomendados por Pérez et al. (15): índice de bondad de ajuste (GFI) $\geq$ ,95; raíz cuadrada media del error de aproximación (RMSEA) < 0,05; e índice de ajuste comparativo $(\mathrm{CFI}) \geq 0,95$. El nivel alfa se determinó antes del análisis en 0,05 . Los análisis se realizaron con ayuda del programa estadístico SPSS de IBM versión 23 y AMOS SPSS.

\section{Aspectos éticos}

Se respetó la confidencialidad de los encuestados, cumpliendo la Declaración de Helsinki. Se informó a cada participante de la voluntariedad de participar en el estudio. Se contó además con la autorización del Comité de Ética de la FMH-USMP. Debido a que la evaluación fue estrictamente confidencial no se pudo obtener los nombres de los alumnos que obtuvieron niveles altos de síntomas depresivos. No obstante, a través de la Central de Tutorías de la FMH-USMP se estuvieron brindando consejerías luego de la aplicación de esta evaluación para que los alumnos puedan acceder a una evaluación posterior voluntaria si lo requerían. 


\section{RESULTADOS}

Respondieron un total de 701 alumnos de medicina, de los cuales se seleccionó de forma aleatoria simple a 200. La edad promedio fue de 20,84 $( \pm 3,142)$ años. La edad mínima fue de 16 y la máxima de 28 años. Las demás características sociodemográficas las podemos encontrar en la tabla 1.

Tabla-1. Características sociodemográficas de 200 estudiantes de medicina de la FMH-USMP.

\begin{tabular}{lc}
\hline Variable & $\mathbf{n}(\mathbf{\%})$ \\
\hline Edad, años, media $( \pm \sigma)$ & $20,84( \pm 3,142)$ \\
[rango] & {$[16-31]$} \\
Género & \\
$\quad$ Masculino & $73(36,5)$ \\
Femenino & $127(63,5)$ \\
Vive con & \\
Acompañado & $179(89,5)$ \\
Solo & $21(10,5)$ \\
Procedencia & \\
Lima & $152(76)$ \\
Provincia & $45(22,5)$ \\
Extranjero & $3(1,5)$ \\
Área de estudios & \\
Ciencias básicas & $105(52,5)$ \\
Clínica & $95(47,5)$ \\
Tratamiento psiquiátrico & \\
Sí & $31(15,5)$ \\
No & $169(84,5)$ \\
\hline
\end{tabular}

б: Desviación estándar

\section{Validez y fiabilidad del PHQ-9}

Para comprobar la pertinencia de realizar un análisis de la validez de la PHQ-9 se calculó la medida de adecuación muestral KMO confirmando dicho resultado con la prueba de esfericidad de Barlett, las cuales fueron satisfactorias (prueba de esfericidad de Bartlett: $x^{2}=917,791, \mathrm{p}<0,001$; medida $\mathrm{KMO}=$ 0,904). El análisis factorial de los 9 ítems de la PHQ-9, a través de una extracción de componentes principales con rotación Varimax y normalización de Kaiser, arrojó un factor con autovalor de 5,079, aportando el $56,43 \%$ de la varianza total. Las cargas factoriales las podemos encontrar en la Tabla 2.

Encontramos que el PHQ-9 tiene una consistencia interna óptima (alfa de Cronbach=0,903). Este valor no aumenta si se elimina algún ítem, todos los cuales tuvieron un índice de homogeneidad $>0,2$.

\section{Análisis factorial confirmatorio}

Mediante el cálculo de la Distancia de Mahalanobis se detectaron 21 casos extremos multivariados, los cuales fueron eliminados antes de seguir con el resto de los análisis. La muestra quedó conformada por 179 casos.

Se comparó los índices de bondad de ajuste de tres modelos (unidimensional, bidimensional y bifactorial) (Figura 1). Los índices de bondad de ajuste del modelo unidimensional y bidimensional fueron menos adecuados en comparación con el modelo bifactorial $\left(x^{2}(17)=26,451, p=0,067 ; \mathrm{CFI}=0,991 ; \mathrm{GFI}=\right.$

Tabla-2. Cargas factoriales del análisis factorial exploratorio del PHQ-9.

\begin{tabular}{lc}
\hline & Factor \\
\hline 2. Se ha sentido decaído (a), deprimido (a), triste o sin esperanzas. & 1 \\
4. Se ha sentido cansado (a) o con poca energía. &, 853 \\
6. Se ha sentido mal con usted mismo (a), o que es un fracaso o que ha quedado mal con usted &, 785 \\
mismo (a) o con su familia. &, 784 \\
7. Ha tenido dificultad para concentrarse en ciertas actividades, tales como leer o ver la televisión. &, 773 \\
5. Sin apetito o ha comido en exceso. &, 757 \\
1. Poco interés o placer en hacer cosas. &, 717 \\
9. Pensamientos de que estaría mejor muerto (a) o de hacerse daño de alguna manera. &, 701 \\
3. Ha tenido dificultad para quedarse o permanecer dormido (a), o ha dormido demasiado. &, 700 \\
8. Se ha movido o hablado tan lento que otras personas podrían haberlo notado o lo contrario, muy &, 675 \\
inquieto(a) o agitado(a) que ha estado moviéndose mucho más de lo normal. &
\end{tabular}

Método de extracción: análisis de componentes principales. 1 factor extraído. 


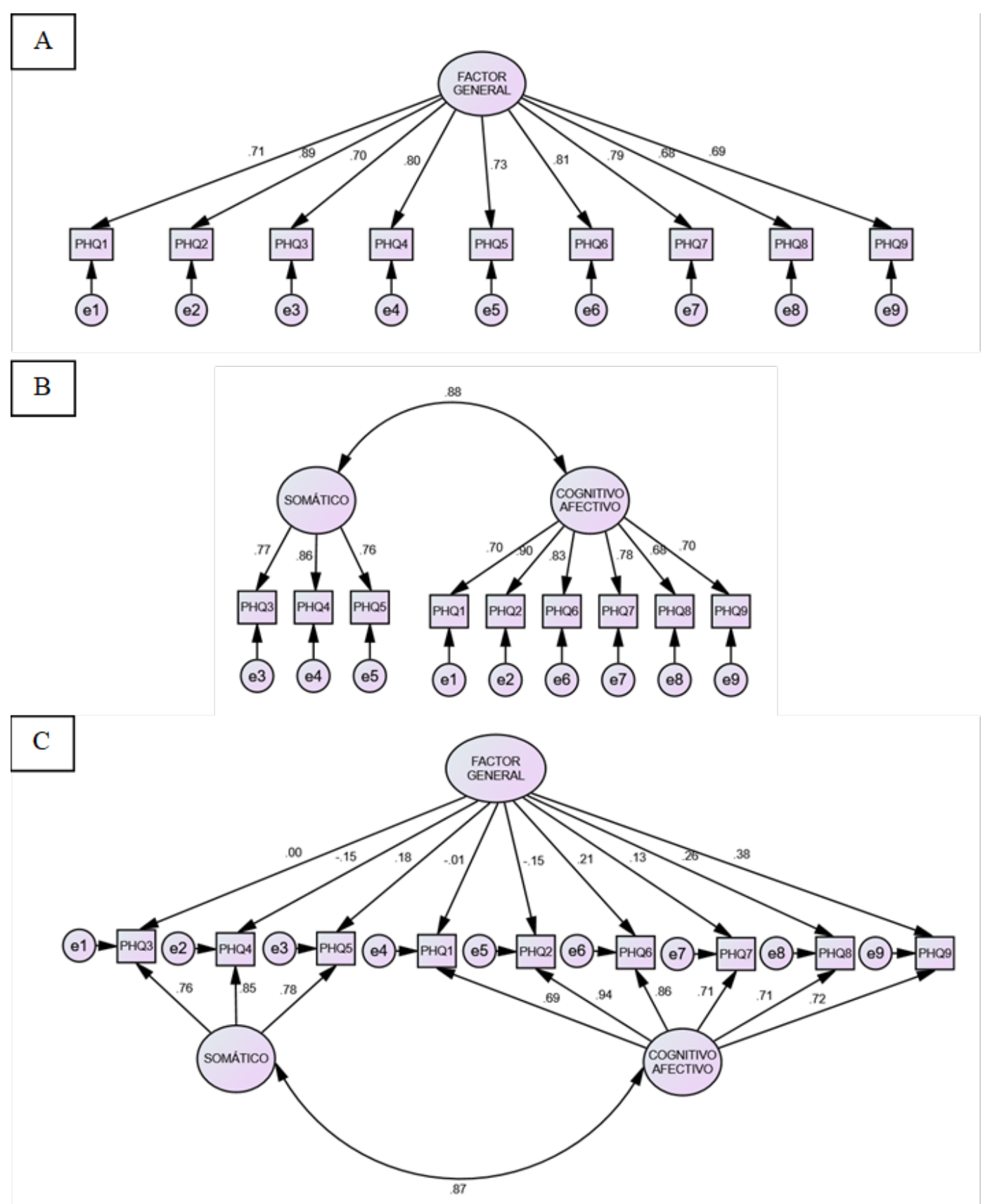

Figura-1. Diagrama del análisis factorial confirmatorio de los ítems del PHQ-9. Las flechas indican las cargas estandarizadas. Modelo unidimensional (A), Modelo bidimensional (B), Modelo bifactorial (C). PHQ1 = anhedonia, PHQ2 = ánimo deprimido, PHQ3 = problemas con el sueño, PHQ4 = fatiga, $\mathrm{PHQ} 5$ = cambios en el apetito, $\mathrm{PHQ6}=$ sentimientos de inutilidad, $\mathrm{PHQ7}=$ problemas con la concentración, PHQ8 = enlentecimiento/agitación, PHQ9 = ideas suicidas.

0,969; RMSEA = 0,056) (Tabla 3). Los coeficientes de regresión estandarizados en el factor somático fueron: Ítem $3=0,758$; Ítem $4=0,848$; Ítem $5=0,775$. En el factor cognitivo/afectivo fueron: Ítem $1=0,689$; Ítem $2=0,936$; Ítem $6=0,860$; Ítem $7=0,714$; Ítem $8=$ 0,712 ; Ítem $9=0,721$.

\section{DISCUSIÓN}

En este estudio se analizaron las propiedades psicométricas del PHQ-9 en estudiantes de medicina de una universidad privada del Perú. Hasta donde tenemos conocimiento, este es el primer estudio del 
Propiedades psicométricas del Patient Health Questionnaire (PHQ-9) en estudiantes de medicina en Lima, Perú.

Tabla-3. Índices de ajuste del modelo unidimensional y bidimensional.

\begin{tabular}{lccccc}
\hline & $\boldsymbol{x}^{\mathbf{2}}(\boldsymbol{p})$ & g.l. & CFI & GFI & RMSEA \\
\hline Modelo unidimensional & $85,129(p=, 000)$ & 27 & 0,942 & 0,900 & 0,110 \\
Modelo bidimensional & $58,476(p=, 000)$ & 26 & 0,968 & 0,930 & 0,084 \\
Modelo bifactorial & $26,451(p=, 067)$ & 17 & 0,991 & 0,969 & 0,056 \\
\hline
\end{tabular}

GFI: índice de bondad de ajuste; RMSEA: raíz cuadrada media del error de aproximación; CFI: índice de ajuste comparativo.

funcionamiento psicométrico del PHQ-9 que se ha realizado en estudiantes de medicina peruanos.

En la muestra estudiada se encontró un comportamiento psicométrico adecuado del PHQ-9, lo cual concuerda con lo reportado en otros estudios $(10,11)$. La consistencia interna que encontramos (alfa de Cronbach $=0,903$ ) fue mayor a la reportada en estudios realizados en estudiantes de medicina de China (alfa de Cronbach $=0,854)(16)$, Corea (alfa de Cronbach $=0,837)(17)$ e India (alfa de Cronbach $=$ $0,835)(18)$.

Si bien, en el AFE se encontró que el PHQ-9 consta de un solo factor, en el AFC no se encontró un buen ajuste de dicho modelo. Por lo que realizamos un AFC en otros dos modelos. Al igual que lo reportado por Doi et al. (12), encontramos que el modelo bifactorial tiene una mejor bondad de ajuste. Esto explicaría los resultados mixtos encontrados en estudios previos que informaron que el PHQ-9 tiene una estructura unidimensional o bidimensional $(12,19,20)$. El modelo bifactorial tiene la ventaja de poder usar tanto una solución unidimensional, es decir, el usar el punto de corte y la puntuación total como una sola variable y una solución bidimensional para evaluar más detalladamente los síntomas depresivos (12). Los hallazgos que reportamos apoyan la idea respecto a que la depresión es un constructo multidimensional, dentro de la cual el ánimo deprimido y los sentimientos de inutilidad son los ítems más importantes, lo que concuerda con un estudio de análisis factorial realizado en estudiantes universitarios colombianos (20). No obstante, algunos investigadores mencionan que debido a la alta correlación entre las dimensiones somática y cognitivo/afectiva, y con la finalidad de garantizar una simplicidad en los modelos, sería recomendable considerar un modelo unidimensional del PHQ-9 (5).

Respecto a los ítems que se encuentran más cercanos al constructo de depresión, encontramos que los ítems 2 = ánimo deprimido, y $6=$ sentimientos de inutilidad, son los más importantes en el factor cognitivo/afectivo. El menos relevante fue el ítem 1 = anhedonia. En el factor somático el ítem más importante fue el 4 = fatiga, mientras que el menos importante fue el 3 = problemas con el sueño. El PHQ-9 tiene ítems que califican con el mismo puntaje síntomas opuestos (insomnio e hipersomnia, retardo y agitación psicomotriz, aumento y disminución del apetito), lo cual podría generar problemas psicométricos.

Este estudio debe ser entendido en sus potenciales limitaciones metodológicas. Primero, estos resultados no pueden ser generalizados a toda la población peruana, ya que solo estudiamos a estudiantes de medicina de una universidad privada. Segundo, no se complementó la información obtenida con el PHQ9 con entrevistas psiquiátricas para confirmar el diagnóstico de depresión.

En conclusión, los resultados obtenidos indican que el PHQ-9 es un instrumento con propiedades psicométricas adecuadas para aplicarse en estudiantes de medicina de una universidad del Perú. El AFC reveló que un modelo bifactorial, en el cual se tome en cuenta tanto el factor general como los factores latentes (somático y cognitivo/afectivo), muestra un mejor ajuste.

Fuentes de financiamiento: El presente estudio ha sido autofinanciado.

Conflicto de intereses: Los autores declaran no tener ningún conflicto de intereses.

\section{Correspondencia:}

\author{
Jeff David Huarcaya Victoria \\ Universidad de San Martín de Porres - Instituto de \\ Investigación \\ Av. Alameda del Corregidor, 1531, La Molina, Lima. \\ Teléfono: (511) 3653640 \\ Correo electrónico: jhuarcayav@usmp.pe
}




\section{REFERENCIAS BIBLIOGRÁFICAS}

1. Instituto Nacional de Salud Mental. Estudio Epidemiológico de Salud Mental en Lima Metropolitana y Callao Replicación 2012: Informe General. Anales de Salud Mental. 2013;29(Supl 1):00.

2. Puthran R, Zhang MW, Tam WW, Ho RC. Prevalence of depression amongst medical students: a metaanalysis. Med Educ. 2016; 50(4):456-68.

3. Kroenke K, Spitzer RL, Williams JB. The PHQ-9: validity of a brief depression severity measure. J Gen Intern Med. 2001; 16(9): 606-13.

4. Spitzer RL, Kroenke K, Williams JB. Validation and utility of a self-report version of PRIME-MD: the PHQ primary care study. Primary Care Evaluation of Mental Disorders. Patient Health Questionnaire. JAMA. 1999;282(18):1737-44.

5. Boothroyd L, Dagnan D, Muncer S. PHQ-9: One factor or two? Psychiatry Res. 2019; 271:532-4.

6. Calderón M, Gálvez-Buccollini JA, Cueva G, Ordoñez C, Bromley C, Fiestas F. Validación de la versión peruana del PHQ-9 para el diagnóstico de depresión. Rev Peru Med Exp Salud Publica. 2012;29:578-579.

7. Zhong Q, Gelaye B, Fann JR, Sanchez SE, Williams MA. Cross-cultural validity of the Spanish version of PHQ-9 among pregnant Peruvian women: a Rasch item response theory analysis. J Affect Disord. 2014;158:148-153.

8. Galarza J. Propiedades psicométricas del Patient Health Questionnaire PHQ-9 Versión Traducida en Pacientes Afectados con Tuberculosis - Lima 20162017. Tesis de Licenciatura en psicología. Lima: Universidad César Vallejo; 2018.

9. Hair J, Black W, Babin BJ, Anderson RE. Multivariate Data Analysis. New Jersey: Essex: Pearson; 2014. p.734.

10. Cassiani-Miranda CA, Vargas-Hernández MC, Pérez-Aníbal E, Herazo-Bustos MI, HernándezCarrillo M. Confiabilidad y dimensión del cuestionario de salud del paciente (PHQ-9) para la detección de síntomas de depresión en estudiantes de ciencias de la salud en Cartagena, 2014. Biomédica. 2017; 37(Supl 1):112-20.
11. Saldivia S, Aslan J, Cova F, Vicente B, Inostroza C, Rincón P. Propiedades psicométricas del PHQ-9 (Patient Health Questionnaire) en centros de atención primaria de Chile. Rev Méd Chile. 2019;147:53-60.

12. Doi S, Ito M, Takebayashi Y, Muramatsu K, Horikoshi M. Factorial validity and invariance of the Patient Health Questionnaire (PHQ)-9 among clinical and non-clinical populations. PLoS One. 2018; 13(7):199235.

13. Boomsma A. Reporting Analyses of Covariance Structures. Struct Equ Model. 2000; 7(3): 461-83.

14. Kline RB. Principles and Practice of Structural Equation Modeling. New York: Guilford Publications; 2011. p.411.

15. Pérez E, Medrano L, Sánchez Rosas J. El Path Analysis: conceptos básicos y ejemplos de aplicación. Revista Argentina de Ciencias del Comportamiento. 2013; 5(1):52-66.

16. Zhang YL, Liang W, Chen ZM, Zhang HM, Zhang JH, Weng XQ, et al. Validity and reliability of Patient Health Questionnaire-9 and Patient Health Questionnaire-2 to screen for depression among college students in China. Asia Pac Psychiatry. 2013;5(4):268-75.

17. Yoon S, Lee Y, Han C, Pae CU, Yoon HK, Patkar AA, et al. Usefulness of the Patient Health Questionnaire-9 for Korean medical students. Acad Psychiatry. 2014; 38(6):661-7.

18. Ganguly S, Samanta M, Roy P, Chatterjee S, Kaplan DW, Basu B. Patient health questionnaire-9 as an effective tool for screening of depression among Indian adolescents. J Adolesc Health. 2013;52(5):54651.

19. Arrieta J, Aguerrebere M, Raviola G, Flores H, Elliott $\mathrm{P}$, Espinosa A, et al. Validity and utility of the patient health questionnaire (PHQ)-2 and PHQ-9 for screening and diagnosis of depression in rural Chiapas, Mexico: A cross-sectional study. J Clin Psychol. 2017;73(9):1076-90.

20. Miranda CAC, Scoppetta O. Factorial structure of the Patient Health Questionnaire-9 as a depression screening instrument for university students in Cartagena, Colombia. Psychiatry Res. 2018;269:4259.

Recibido: $22 / 01 / 2020$

Aceptado: 13/05/2020 